EUROPEAN ORGANISATION FOR NUCLEAR RESEARCH

CERN-PPE/96-121

August 26, 1996

\title{
Measurement of the Branching Fraction of the Radiative Decay $\tau^{-} \rightarrow \mu^{-} \bar{\nu}_{\mu} \nu_{\tau} \gamma$
}

\author{
The OPAL Collaboration
}

\begin{abstract}
The branching ratio of the radiative $\tau$ decay $\tau^{-} \rightarrow \mu^{-} \bar{\nu}_{\mu} \nu_{\tau} \gamma$ has been measured using data collected with the OPAL detector at LEP. The result is $\mathrm{B}\left(\tau^{-} \rightarrow \mu^{-} \bar{\nu}_{\mu} \nu_{\tau} \gamma\right)=(3.0 \pm 0.4 \pm 0.5) \times 10^{-3}$ for photon energies above $20 \mathrm{MeV}$ measured in the $\tau$ rest frame. The result is in good agreement with the Standard Model expectation.
\end{abstract}

(To be submitted to Physics Letters B) 


\section{The OPAL Collaboration}

G. Alexander ${ }^{23}$, J. Allison ${ }^{16}$, N. Altekamp ${ }^{5}$, K. Ametewee ${ }^{25}$, K.J. Anderson ${ }^{9}$, S. Anderson ${ }^{12}$, S. Arcelli ${ }^{2}$, S. Asai ${ }^{24}$, D. Axen ${ }^{29}$, G. Azuelos ${ }^{18, a}$, A.H. Ball ${ }^{17}$, E. Barberio ${ }^{8}$, R.J. Barlow ${ }^{16}$, R. Bartoldus ${ }^{3}$, J.R. Batley ${ }^{5}$, J. Bechtluft ${ }^{14}$, C. Beeston ${ }^{16}$, T. Behnke ${ }^{8}$, A.N. Bell ${ }^{1}$, K.W. Bell ${ }^{20}$, G. Bella ${ }^{23}$, S. Bentvelsen ${ }^{8}$, P. Berlich ${ }^{10}$, S. Bethke ${ }^{14}$, O. Biebel ${ }^{14}$, V. Blobel ${ }^{8}$, I.J. Bloodworth ${ }^{1}$, J.E. Bloomer ${ }^{1}$, M. Bobinski ${ }^{10}$, P. Bock ${ }^{11}$, H.M. Bosch ${ }^{11}$, M. Boutemeur ${ }^{34}$, B.T. Bouwens ${ }^{12}$, S. Braibant ${ }^{12}$, R.M. Brown ${ }^{20}$, H.J. Burckhart ${ }^{8}$, C. Burgard ${ }^{8}$, R. Bürgin ${ }^{10}$, P. Capiluppi ${ }^{2}$, R.K. Carnegie ${ }^{6}$, A.A. Carter ${ }^{13}$, J.R. Carter $^{5}$, C.Y. Chang ${ }^{17}$, C. Charlesworth ${ }^{6}$, D.G. Charlton ${ }^{1, b}$, D. Chrisman ${ }^{4}$, S.L. Chu ${ }^{4}$, P.E.L. Clarke ${ }^{15}$, I. Cohen ${ }^{23}$, J.E. Conboy ${ }^{15}$, O.C. Cooke ${ }^{16}$, M. Cuffiani ${ }^{2}$, S. Dado ${ }^{22}$, C. Dallapiccola ${ }^{17}$, G.M. Dallavalle ${ }^{2}$, S. De Jong ${ }^{12}$, L.A. del Pozo ${ }^{8}$, K. Desch ${ }^{3}$, M.S. Dixit ${ }^{7}$, E. do Couto e Silva ${ }^{12}$, M. Doucet ${ }^{18}$, E. Duchovni ${ }^{26}$, G. Duckeck ${ }^{34}$, I.P. Duerdoth ${ }^{16}$, J.E.G. Edwards ${ }^{16}$, P.G. Estabrooks ${ }^{6}$, H.G. Evans ${ }^{9}$, M. Evans ${ }^{13}$, F. Fabbri ${ }^{2}$, P. Fath ${ }^{11}$, F. Fiedler ${ }^{12}$, M. Fierro ${ }^{2}$, H.M. Fischer ${ }^{3}$, R. Folman ${ }^{26}$, D.G. Fong ${ }^{17}$, M. Foucher ${ }^{17}$, A. Fürtjes ${ }^{8}$, P. Gagnon ${ }^{7}$, A. Gaidot ${ }^{21}$, J.W. Gary ${ }^{4}$, J. Gascon ${ }^{18}$, S.M. Gascon-Shotkin ${ }^{17}$, N.I. Geddes ${ }^{20}$, C. Geich-Gimbel ${ }^{3}$, F.X. Gentit ${ }^{21}$, T. Geralis ${ }^{20}$, G. Giacomelli ${ }^{2}$, P. Giacomelli ${ }^{4}$, R. Giacomelli ${ }^{2}$, V. Gibson ${ }^{5}$, W.R. Gibson ${ }^{13}$, D.M. Gingrich ${ }^{30, a}$, D. Glenzinski ${ }^{9}$, J. Goldberg ${ }^{22}$, M.J. Goodrick ${ }^{5}$, W. Gorn ${ }^{4}$, C. Grandi ${ }^{2}$, E. Gross ${ }^{26}$, M. Gruwé ${ }^{8}$, C. Hajdu ${ }^{32}$, G.G. Hanson ${ }^{12}$, M. Hansroul ${ }^{8}$, M. Hapke ${ }^{13}$, C.K. Hargrove ${ }^{7}$, P.A. Hart ${ }^{9}$, C. Hartmann ${ }^{3}$, M. Hauschild ${ }^{8}$, C.M.Hawkes ${ }^{5}$, R. Hawkings ${ }^{8}$, R.J. Hemingway ${ }^{6}$, G. Herten ${ }^{10}$, R.D. Heuer ${ }^{8}$, M.D. Hildreth ${ }^{8}$, J.C. Hill ${ }^{5}$, S.J. Hillier ${ }^{1}$, T. Hilse ${ }^{10}$, P.R. Hobson ${ }^{25}$, R.J.Homer ${ }^{1}$, A.K. Honma ${ }^{28, a}$, D. Horváth ${ }^{32, c}$, R. Howard ${ }^{29}$, R.E. Hughes-Jones ${ }^{16}$, D.E. Hutchcroft ${ }^{5}$, P. Igo-Kemenes ${ }^{11}$, D.C. Imrie ${ }^{25}$,

M.R. Ingram ${ }^{16}$, K. Ishii ${ }^{24}$, A. Jawahery ${ }^{17}$, P.W. Jeffreys ${ }^{20}$, H. Jeremie ${ }^{18}$, M. Jimack ${ }^{1}$, A. Joly ${ }^{18}$, C.R. Jones ${ }^{5}$, G. Jones ${ }^{16}$, M. Jones ${ }^{6}$, R.W.L. Jones ${ }^{8}$, U. Jost ${ }^{11}$, P. Jovanovic ${ }^{1}$, T.R. Junk ${ }^{8}$, D. Karlen ${ }^{6}$, K. Kawagoe ${ }^{24}$, T. Kawamoto ${ }^{24}$, R.K. Keeler ${ }^{28}$, R.G. Kellogg ${ }^{17}$, B.W. Kennedy ${ }^{20}$, B.J. King ${ }^{8}$, J. Kirk ${ }^{29}$, S. Kluth ${ }^{8}$, T. Kobayashi ${ }^{24}$, M. Kobel ${ }^{10}$, D.S. Koetke ${ }^{6}$, T.P. Kokott ${ }^{3}$, M. Kolrep ${ }^{10}$, S. Komamiya ${ }^{24}$, R. Kowalewski ${ }^{8}$, T. Kress ${ }^{11}$, P. Krieger ${ }^{6}$, J. von Krogh ${ }^{11}$, P. Kyberd ${ }^{13}$, G.D. Lafferty ${ }^{16}$, H. Lafoux ${ }^{21}$, R. Lahmann ${ }^{17}$, W.P. Lai ${ }^{19}$, D. Lanske ${ }^{14}$, J. Lauber ${ }^{15}$, S.R. Lautenschlager ${ }^{31}$, J.G. Layter ${ }^{4}$, D. Lazic ${ }^{22}$, A.M. Lee ${ }^{31}$, E. Lefebvre ${ }^{18}$, D. Lellouch ${ }^{26}$, J. Letts ${ }^{2}$, L. Levinson ${ }^{26}$, C. Lewis ${ }^{15}$, S.L. Lloyd ${ }^{13}$, F.K. Loebinger ${ }^{16}$, G.D. Long ${ }^{17}$, M.J. Losty ${ }^{7}$, J. Ludwig ${ }^{10}$, A. Malik ${ }^{21}$, M. Mannelli ${ }^{8}$, S. Marcellini ${ }^{2}$, C. Markus ${ }^{3}$, A.J. Martin ${ }^{13}$, J.P. Martin ${ }^{18}$,

G. Martinez ${ }^{17}$, T. Mashimo ${ }^{24}$, W. Matthews ${ }^{25}$, P. Mättig ${ }^{3}$, W.J. McDonald ${ }^{30}$, J. McKenna ${ }^{29}$,

E.A.Mckigney ${ }^{15}$, T.J. McMahon ${ }^{1}$, A.I. McNab ${ }^{13}$, R.A.McPherson ${ }^{8}$, F. Meijers ${ }^{8}$, S. Menke ${ }^{3}$,

F.S. Merritt ${ }^{9}$, H. Mes ${ }^{7}$, J. Meyer ${ }^{27}$, A. Michelini' ${ }^{2}$, G. Mikenberg ${ }^{26}$, D.J. Miller ${ }^{15}$, R. Mir ${ }^{26}$, W. Mohr ${ }^{10}$, A. Montanari ${ }^{2}$, T. Mori ${ }^{24}$, M. Morii ${ }^{24}$, U. Müller ${ }^{3}$, K. Nagai ${ }^{26}$, I. Nakamura ${ }^{24}$, H.A. Neal ${ }^{8}$, B. Nellen ${ }^{3}$, B. Nijjhar ${ }^{16}$, R. Nisius ${ }^{8}$, S.W. O'Neale ${ }^{1}$, F.G.Oakham ${ }^{7}$, F. Odorici ${ }^{2}$, H.O. Ogren ${ }^{12}$, T.Omori ${ }^{24}$, M.J. Oreglia ${ }^{9}$, S. Orito ${ }^{24}$, J. Pálinkás ${ }^{33, d}$, G. Pásztor ${ }^{32}$, J.R. Pater ${ }^{16}$, G.N.Patrick ${ }^{20}$, J. Patt ${ }^{10}$, M.J. Pearce ${ }^{1}$, S. Petzold ${ }^{27}$, P. Pfeifenschneider ${ }^{14}$, J.E. Pilcher ${ }^{9}$, J. Pinfold ${ }^{30}$, D.E. Plane ${ }^{8}$, P. Poffenberger ${ }^{28}$, B. Poli ${ }^{2}$, A. Posthaus ${ }^{3}$, H. Przysiezniak ${ }^{30}$, D.L. Rees ${ }^{1}$, D. Rigby ${ }^{1}$, S.A. Robins ${ }^{13}$, N. Rodning ${ }^{30}$, J.M. Roney ${ }^{28}$, A. Rooke $^{15}$, E. Ros ${ }^{8}$, A.M. Rossi ${ }^{2}$, M. Rosvick ${ }^{28}$, P. Routenburg ${ }^{30}$, Y. Rozen ${ }^{22}$, K. Runge ${ }^{10}$, O. Runolfsson ${ }^{8}$, U. Ruppel ${ }^{14}$, D.R. Rust ${ }^{12}$, R. Rylko ${ }^{25}$, K. Sachs ${ }^{10}$, E.K.G.Sarkisyan ${ }^{23}$, M.Sasaki ${ }^{24}$, C.Sbarra ${ }^{2}$,

A.D.Schaile ${ }^{34}$, O.Schaile ${ }^{34}$, F. Scharf ${ }^{3}$, P.Scharff-Hansen ${ }^{8}$, P.Schenk ${ }^{4}$, B.Schmitt ${ }^{8}$, S.Schmitt ${ }^{11}$, M.Schröder ${ }^{8}$, H.C.Schultz-Coulon ${ }^{10}$, M.Schulz ${ }^{8}$, M.Schumacher ${ }^{3}$, P.Schütz ${ }^{3}$, W.G.Scott ${ }^{20}$, T.G.Shears ${ }^{16}$, B.C.Shen ${ }^{4}$, C.H.Shepherd-Themistocleous ${ }^{27}$, P. Sherwood ${ }^{15}$, G.P.Siroli ${ }^{2}$, A.Sittler ${ }^{27}$, A.Skillman ${ }^{15}$, A.Skuja ${ }^{17}$, A.M.Smith ${ }^{8}$, T.J.Smith ${ }^{28}$, G.A.Snow ${ }^{17}$, R.Sobie ${ }^{28}$, S.Söldner-Rembold ${ }^{10}$, R.W.Springer ${ }^{30}$, M.Sproston ${ }^{20}$, A.Stahl ${ }^{3}$, M.Starks ${ }^{12}$, 
M.Steiert ${ }^{11}$, K.Stephens ${ }^{16}$, J.Steuerer ${ }^{27}$, B. Stockhausen ${ }^{3}$, D. Strom ${ }^{19}$, F. Strumia ${ }^{8}$, P. Szymanski ${ }^{20}$, R. Tafirout ${ }^{18}$, S.D. Talbot ${ }^{1}$, S. Tanaka ${ }^{24}$, P. Taras $^{18}$, S. Tarem ${ }^{22}$, M. Tecchio ${ }^{8}$,

M. Thiergen ${ }^{10}$, M.A. Thomson ${ }^{8}$, E. von Törne ${ }^{3}$, S. Towers ${ }^{6}$, T. Tsukamoto ${ }^{24}$, E. Tsur ${ }^{23}$, A.S. Turcot ${ }^{9}$, M.F. Turner-Watson ${ }^{8}$, P. Utzat ${ }^{11}$, R. Van Kooten ${ }^{12}$, G. Vasseur ${ }^{21}$, M. Verzocchi ${ }^{10}$, P. Vikas ${ }^{18}$, M. Vincter ${ }^{28}$, E.H. Vokurka ${ }^{16}$, H.H. $\operatorname{Voss}^{3}$, F. Wäckerle ${ }^{10}$, A. Wagner ${ }^{27}$, C.P. Ward ${ }^{5}$, D.R. Ward ${ }^{5}$, J.J. Ward ${ }^{15}$, P.M. Watkins ${ }^{1}$, A.T. Watson ${ }^{1}$, N.K. Watson ${ }^{7}$, P. Weber ${ }^{6}$, P.S. Wells ${ }^{8}$, N. Wermes ${ }^{3}$, J.S. White ${ }^{28}$, B. Wilkens ${ }^{10}$, G.W.Wilson ${ }^{27}$, J.A. Wilson ${ }^{1}$, G. Wolf ${ }^{26}$, S. Wotton ${ }^{5}$, T.R. Wyatt ${ }^{16}$, S. Yamashita ${ }^{24}$, G. Yekutieli ${ }^{26}$, V. Zacek $^{18}$,

${ }^{1}$ School of Physics and Space Research, University of Birmingham, Birmingham B15 2TT, UK ${ }^{2}$ Dipartimento di Fisica dell' Università di Bologna and INFN, I-40126 Bologna, Italy

${ }^{3}$ Physikalisches Institut, Universität Bonn, D-53115 Bonn, Germany

${ }^{4}$ Department of Physics, University of California, Riverside CA 92521, USA

${ }^{5}$ Cavendish Laboratory, Cambridge CB3 0HE, UK

${ }^{6}$ Ottawa-Carleton Institute for Physics, Department of Physics, Carleton University, Ottawa, Ontario K1S 5B6, Canada

${ }^{7}$ Centre for Research in Particle Physics, Carleton University, Ottawa, Ontario K1S 5B6, Canada

${ }^{8}$ CERN, European Organisation for Particle Physics, CH-1211 Geneva 23, Switzerland

${ }^{9}$ Enrico Fermi Institute and Department of Physics, University of Chicago, Chicago IL 60637 , USA

${ }^{10}$ Fakultät für Physik, Albert Ludwigs Universität, D-79104 Freiburg, Germany

${ }^{11}$ Physikalisches Institut, Universität Heidelberg, D-69120 Heidelberg, Germany

${ }^{12}$ Indiana University, Department of Physics, Swain Hall West 117, Bloomington IN 47405, USA

${ }^{13}$ Queen Mary and Westfield College, University of London, London E1 4NS, UK

${ }^{14}$ Technische Hochschule Aachen, III Physikalisches Institut, Sommerfeldstrasse 26-28, D-52056

Aachen, Germany

${ }^{15}$ University College London, London WC1E 6BT, UK

${ }^{16}$ Department of Physics, Schuster Laboratory, The University, Manchester M13 9PL, UK

${ }^{17}$ Department of Physics, University of Maryland, College Park, MD 20742, USA

${ }^{18}$ Laboratoire de Physique Nucléaire, Université de Montréal, Montréal, Quebec H3C 3J7, Canada

${ }^{19}$ University of Oregon, Department of Physics, Eugene OR 97403, USA

${ }^{20}$ Rutherford Appleton Laboratory, Chilton, Didcot, Oxfordshire OX11 0QX, UK

${ }^{21}$ CEA, DAPNIA/SPP, CE-Saclay, F-91191 Gif-sur-Yvette, France

${ }^{22}$ Department of Physics, Technion-Israel Institute of Technology, Haifa 32000, Israel

${ }^{23}$ Department of Physics and Astronomy, Tel Aviv University, Tel Aviv 69978, Israel

${ }^{24}$ International Centre for Elementary Particle Physics and Department of Physics, University of Tokyo, Tokyo 113, and Kobe University, Kobe 657, Japan

${ }^{25}$ Brunel University, Uxbridge, Middlesex UB8 3PH, UK

${ }^{26}$ Particle Physics Department, Weizmann Institute of Science, Rehovot 76100, Israel

${ }^{27}$ Universität Hamburg/DESY, II Institut für Experimental Physik, Notkestrasse 85, D-22607 Hamburg, Germany

${ }^{28}$ University of Victoria, Department of Physics, P O Box 3055, Victoria BC V8W 3P6, Canada

${ }^{29}$ University of British Columbia, Department of Physics, Vancouver BC V6T 1Z1, Canada 
${ }^{30}$ University of Alberta, Department of Physics, Edmonton AB T6G 2J1, Canada

${ }^{31}$ Duke University, Dept of Physics, Durham, NC 27708-0305, USA

${ }^{32}$ Research Institute for Particle and Nuclear Physics, H-1525 Budapest, P O Box 49, Hungary ${ }^{33}$ Institute of Nuclear Research, H-4001 Debrecen, P O Box 51, Hungary

${ }^{34}$ Ludwigs-Maximilians-Universität München, Sektion Physik, Am Coulombwall 1, D-85748 Garching, Germany

${ }^{a}$ and at TRIUMF, Vancouver, Canada V6T $2 \mathrm{~A} 3$

${ }^{b}$ and Royal Society University Research Fellow

${ }^{c}$ and Institute of Nuclear Research, Debrecen, Hungary

${ }^{d}$ and Department of Experimental Physics, Lajos Kossuth University, Debrecen, Hungary 


\section{Introduction}

The leptonic branching fractions of $\tau$ lepton decays have been precisely measured by many experiments $[1,2,3]$. These measurements, together with the measurements of the mass of the $\tau$ lepton and its lifetime, constitute an important test of the Standard Model and provide evidence for the universality of the electroweak lepton couplings [4]. The decay ${ }^{1} \tau^{-} \rightarrow l^{-} \bar{\nu}_{l} \nu_{\tau} \gamma$, where $l=e, \mu$, involves radiative corrections to the $\tau^{-} \rightarrow l^{-} \bar{\nu}_{l} \nu_{\tau}$ decay. The differential decay rate for the process $\tau^{-} \rightarrow \mu^{-} \bar{\nu}_{\mu} \nu_{\tau} \gamma$ can be obtained from the expression for the analogous decay $\mu^{-} \rightarrow \mathrm{e}^{-} \bar{\nu}_{\mathrm{e}} \nu_{\tau} \gamma$ which has been calculated by several authors [5]. The photon in the final state is sensitive to right-handed couplings of the daughter lepton in the decay [6,7]. In the present measurement, limited statistics do not permit a measurement of couplings outside the Standard Model. Therefore, we restrict ourselves to comparing the integrated branching fraction with the value that emerges from the standard event simulation Monte Carlo (MC) programs [8, 9, 10], which include the theoretical description of this decay.

Within the Standard Model the radiative decay of the $\tau$ to a muon, two neutrinos, and a photon is described at the tree level by the two diagrams shown in fig. 1. A third process, with a photon radiating from the intermediate $W$, is not shown because it is strongly suppressed by a factor involving the $\mathrm{W}$ mass. Final-state radiation (FSR) in the $\tau$-pair production process

a)

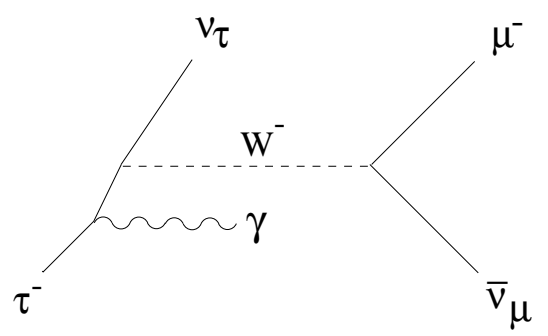

b)

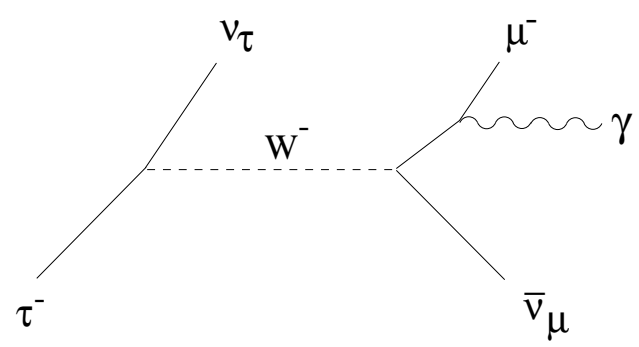

Figure 1: (a) and (b) show processes contributing to the radiative muonic $\tau$ decay, $\tau^{-} \rightarrow$ $\mu^{-} \bar{\nu}_{\mu} \nu_{\tau} \gamma$

$\mathrm{e}^{+} \mathrm{e}^{-} \rightarrow \tau^{+} \tau^{-} \gamma$, in which the photon does not originate from the $\tau$ decay process itself, is considered as background in this analysis.

The first measurement of the radiative branching fraction in leptonic $\tau$ decays was performed by MARK II [11] at an $\mathrm{e}^{+} \mathrm{e}^{-}$centre-of-mass energy of $29 \mathrm{GeV}$. The result was $B\left(\tau^{-} \rightarrow \mu^{-} \bar{\nu}_{\mu} \nu_{\tau} \gamma\right)=$ $(2.3 \pm 1.1) \times 10^{-3}$ for $E_{\gamma}^{*}>37 \mathrm{MeV}$, where $E_{\gamma}^{*}$ is the photon energy in the $\tau$ rest frame. The photon energy spectrum rises steeply for small photon energies. Therefore any measurement of the radiative branching fraction has to be made above some threshold energy in the $\tau$ rest frame. Here we report on the first measurement of $\tau^{-} \rightarrow \mu^{-} \bar{\nu}_{\mu} \nu_{\tau} \gamma$ obtained at LEP, for photon energies $E_{\gamma}^{*}>20 \mathrm{MeV}$, using data collected with the OPAL detector in the years 1991 to 1994 , corresponding to an integrated luminosity of $130 \mathrm{pb}^{-1}$.

\footnotetext{
${ }^{1}$ References in this paper to specific charge states apply to the charge conjugate states as well.
} 


\section{Experimental Considerations}

The OPAL detector at LEP is described in detail elsewhere [12]. The detector components important for this analysis are briefly mentioned here. Tracking is performed by the central tracking chambers which consist of a silicon microvertex detector surrounding the beam pipe, a precision vertex drift chamber covering radii from $10 \mathrm{~cm}$ to $21 \mathrm{~cm}$, followed by the jet chamber which extends to a radius of $185 \mathrm{~cm}$. Precise measurements of the $z$-coordinate ${ }^{2}$ are provided by stereo wires of the vertex drift chamber and by the $z$-chambers located outside the jet chamber in the region $|\cos \theta| \leq 0.72$. A $0.435 \mathrm{~T}$ axial magnetic field is provided by a solenoid. Outside the magnet coil is the electromagnetic calorimeter which in the barrel region consists of 9440 lead glass blocks of $10 \times 10 \mathrm{~cm}^{2}$ front cross section and a depth of 24.6 radiation lengths. Each block covers an angular region of $\sim 2.3^{\circ}$ in $\theta$ and in $\phi$, approximately pointing towards the interaction region. The non-pointing endcaps were not used in this analysis. In front of the electromagnetic calorimeter in the barrel lies a time-of-flight detector and a preshower system (presampler) consisting of limited streamer tubes. The hadronic calorimeter, which lies outside the electromagnetic calorimeter, is a sampling device with up to 9 layers of limited streamer tubes instrumenting the iron of the magnet return yoke. Muon chambers are located outside the hadron calorimeter.

At centre-of-mass energies of $91 \mathrm{GeV} \tau$ leptons from $\mathrm{e}^{+} \mathrm{e}^{-} \rightarrow \tau^{+} \tau^{-}$receive a large Lorentz boost and hence decay into two narrow, low multiplicity jets, which are almost back to back. Therefore most photons radiated in leptonic $\tau$ decays lie very close to the charged lepton creating only a single cluster of energy deposition in the electromagnetic calorimeter (ECAL). Only in the rare case when the photon is emitted at a relatively large angle with respect to the flight direction of the daughter lepton can more than one ECAL cluster be resolved. Muons leave a relatively small energy deposit in the ECAL, typically $400 \mathrm{MeV}$, which is largely independent of the muon momentum. Hence radiative decays, $\tau^{-} \rightarrow \mu^{-} \bar{\nu}_{\mu} \nu_{\tau} \gamma$, can generally be identified only by reconstructing the photon from the energy deposit in the ECAL cluster associated with the muon track. The branching fraction strongly depends on the minimum photon energy chosen in the selection. Because of this dependence, the knowledge of the reconstructed photon energy from the overlapping muon and photon cluster will be the main systematic uncertainty in the analysis.

In addition to the selection of $\tau^{-} \rightarrow \mu^{-} \bar{\nu}_{\mu} \nu_{\tau}(\gamma)$ candidate events, the number of background events, the selection efficiency, and the total number of produced $\tau$ pairs are needed in order to calculate the branching fraction. The important backgrounds for this study come from nonminimum ionizing interactions of muons in the ECAL, which we refer to as "muon interactions", giving rise to fake photon candidates, and from residual hadronic $\tau$ decay background in the sample. The background, scaled to the number of produced $\tau$ pairs, and the selection efficiency are obtained from Monte Carlo simulation, and checked against control samples in the data.

\footnotetext{
${ }^{2}$ The $z$-coordinate is defined along the $\mathrm{e}^{-}$-beam direction, from which the polar angle $\theta$ is measured. The azimuth $\phi$ is defined by having $x$ pointing to the centre of LEP and $y$ upwards.
} 


\section{Event Selection}

The data used in this analysis are selected using a three step procedure. A sample of $\mathrm{e}^{+} \mathrm{e}^{-} \rightarrow$ $\tau^{+} \tau^{-}$events is selected first, followed by the identification of muonic $\tau$ decays ${ }^{3}, \tau^{-} \rightarrow \mu^{-} \bar{\nu}_{\mu} \nu_{\tau}(\gamma)$. Finally, in a third step, radiative $\tau^{-} \rightarrow \mu^{-} \bar{\nu}_{\mu} \nu_{\tau} \gamma$ decays are identified by reconstructing the photon.

In the first step, the OPAL standard $\tau$ selection [13], all tracks and electromagnetic clusters within a cone of $35^{\circ}$ half opening angle are grouped together and considered to be the decay products of one $\tau$ lepton. The selection requires exactly two such cones with at least one good track in each cone. Discrimination against multihadronic background is obtained via cuts on the number of tracks and clusters in the event. Background from cosmic rays is suppressed mainly by requirements on the time-of-flight signal. Cuts on the visible energy and the acollinearity of the cones remove background from two-photon events. In order to remove background from Bhabha scattering and $\mu$ pair events additional cuts are applied on the momenta and the energy deposited in the electromagnetic calorimeter as well as on signals from the muon chambers. A sample of $74769 \tau$ pair candidate events in the barrel region of the detector, $|\cos \theta|<0.68$, results from applying this selection to the 1991-1994 data sample. The background in this sample from other types of events is estimated from Monte Carlo to be $(1.70 \pm 0.12) \%$ [3].

In the second step candidates for muonic $\tau$ decays are selected from the $\mathrm{e}^{+} \mathrm{e}^{-} \rightarrow \tau^{+} \tau^{-}$ sample using a channel likelihood technique. Probabilities for an event to belong to a specific decay mode are calculated using several variables, comparing the measured values with reference distributions generated by Monte Carlo. The probabilities for the different variables are combined in a likelihood value for each decay channel. A more detailed account of this technique is described in a previous publication [14]. In this analysis four detector variables are used in the likelihood selection:

- the specific ionization energy loss, $\mathrm{d} E / \mathrm{d} x$, measured for a track in the central jet chamber,

- the total number of hit layers in the outer three layers of the hadron calorimeter and the layers of the muon chambers,

- the average number of hits per hit layer in the hadron calorimeter,

- the logarithm of the $\chi^{2}$ probability for the matching of a track segment in the muon chamber with the extrapolated track from the jet chamber.

The $\mathrm{d} E / \mathrm{d} x$ expectation value for different decay channels, used in the first variable, is calculated using a parametrization of the Bethe-Bloch formula including corrections specific to the respective track environment [15]. After normalizing the sum of the likelihood values for all channels to unity, a likelihood value for the muonic decay of at least 0.92 is demanded for a $\tau$ decay to be classified as $\tau^{-} \rightarrow \mu^{-} \bar{\nu}_{\mu} \nu_{\tau}(\gamma)$. The likelihood distribution for muonic $\tau$ decays in the $\tau$ event sample is shown in fig. 2. A total of $20926 \tau^{-} \rightarrow \mu^{-} \bar{\nu}_{\mu} \nu_{\tau}(\gamma)$ candidates are selected by this likelihood technique with an estimated background of $2.5 \%$. The variables used in the

\footnotetext{
${ }^{3} \mathrm{By} \tau^{-} \rightarrow \mu^{-} \bar{\nu}_{\mu} \nu_{\tau}(\gamma)$ we denote both decay channels: $\tau^{-} \rightarrow \mu^{-} \bar{\nu}_{\mu} \nu_{\tau}$ and $\tau^{-} \rightarrow \mu^{-} \bar{\nu}_{\mu} \nu_{\tau} \gamma$
} 


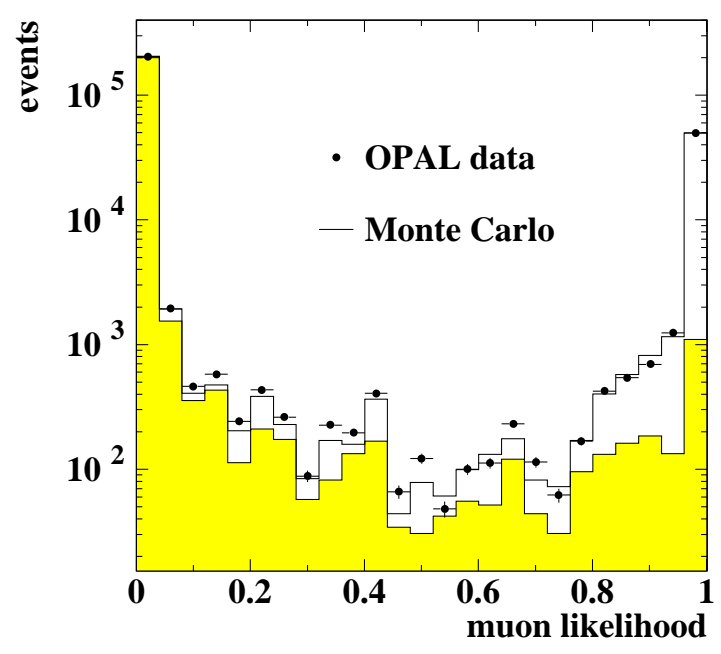

Figure 2: The figure shows the muon likelihood distribution for the selected $\tau$ decays. The histogram is the Monte Carlo prediction in which the shaded part corresponds to background from other $\tau$ decays, while the points correspond to the data.

likelihood do not use ECAL or presampler information because this would bias the radiative fraction in muonic $\tau$ decays.

In the third step, muonic $\tau$ decay candidates with photon radiation are selected by reconstructing a photon in the ECAL. Because photon and muon clusters have a high probability to overlap, the average energy deposit of a single muon is subtracted from the lead glass block associated with the muon track in the jet chamber and then the cluster algorithm is re-run. Fig. 3(a) shows the distribution of energy deposited in the ECAL by single muons from $\mathrm{e}^{+} \mathrm{e}^{-} \rightarrow \mu^{+} \mu^{-}$ events for both the data and Monte Carlo. For this study, $\mathrm{e}^{+} \mathrm{e}^{-} \rightarrow \mu^{+} \mu^{-}$events without photon radiation have been selected using the $\mu$-pair selection described in [13] and demanding a small acollinearity angle $\left(<0.05^{\circ}\right)$ of the muons as well as high muon momenta $(>44.5 \mathrm{GeV})$. The average energy deposit in the data is $420 \mathrm{MeV}$ with a spread (rms) of $\sim 150 \mathrm{MeV}$, considering energy depositions up to $1 \mathrm{GeV}$. The corresponding average in the Monte Carlo simulation is about $25 \mathrm{MeV}$ higher and the spread is about $20 \mathrm{MeV}$ less. Hence different average muon ECAL energy deposits are subtracted in data and Monte Carlo in order to reconstruct the photon. Systematic uncertainties in the reconstructed energy and background due to the different spectra for the observed energy deposition of muons in data and Monte Carlo are included in the systematic studies of background and efficiency described below. The energy deposit is in most cases restricted to one ECAL block only and it is largely independent of the muon momentum as demonstrated by fig. 3(b), which shows the same quantities as fig. 3(a) but for low energy muons taken from $\tau^{-} \rightarrow \mu^{-} \bar{\nu}_{\mu} \nu_{\tau}$ events.

Only events with two or fewer ECAL clusters in the cone of the considered $\tau$ decay are allowed for the photon reconstruction because events with more clusters have a high probability of originating from background processes. If there is just one cluster remaining in the cone after the average muon ECAL energy deposit has been removed, it is considered to be a photon. If two separate clusters remain, the cluster which is closer to the charged track is assumed to 

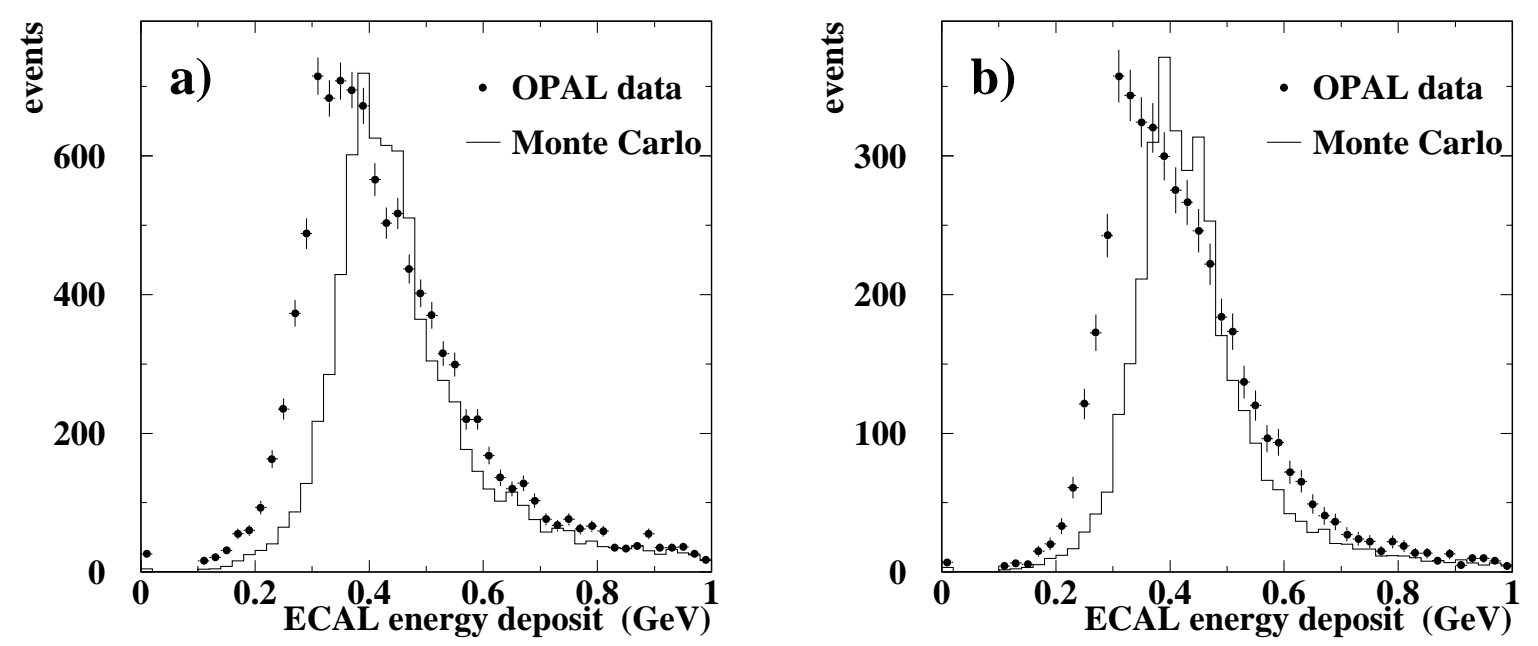

Figure 3: (a) and (b) show the energy deposit of muons in the ECAL; the points with error bars are taken from the data while the histograms show the Monte Carlo estimate. (a) is taken from $\mu$ pair events (45GeV) and (b) uses muons from $\tau^{-} \rightarrow \mu^{-} \bar{\nu}_{\mu} \nu_{\tau}$ decays with less than $30 \mathrm{GeV}$ energy.

result from a larger than average energy deposition by the muon. Hence the cluster which lies further from the muon track is assumed to be due to the photon. An exception is made to this in the case where the cluster closer to the muon is more energetic than the secondary cluster and has an energy greater than $1.7 \mathrm{GeV}$. In this case, the secondary cluster is ignored and the closer cluster is designated the photon candidate. This exception was found to be necessary from Monte Carlo studies in order to be less sensitive to secondary clusters originating from shower fluctuations.

Background to radiative $\tau$ decay is expected from physics processes with photon radiation from other sources, most notably final state radiation from the $\tau, \mu$ bremsstrahlung in the material in front of the ECAL and $\mu$ pair production in $\mathrm{e}^{+} \mathrm{e}^{-}$as well as in two-photon annihilation processes with FSR. Other sources of background arise from fake photons caused by muons "interacting" in the ECAL, i.e. depositing much more than the average energy, and misclassified hadronic $\tau$ decays, especially those with $\pi^{0}$ 's in the final state. The various background sources are listed in table 1 and are discussed further below. Additional cuts on ECAL variables are applied in order to reduce these backgrounds:

- A minimum of $1 \mathrm{GeV}$ is demanded for the reconstructed photon. This minimum energy is necessary in order to separate real photons from shower fluctuations of the muon energy deposit in the ECAL.

- Background from fake photons caused by muons that interact in the ECAL and deposit much more than the average energy is further reduced by requiring that the number of ECAL blocks in the cluster is at least three and that the fraction of the cluster energy in the most energetic block is less than $90 \%$. These background events look similar to 
$\tau^{-} \rightarrow \mu^{-} \bar{\nu}_{\mu} \nu_{\tau} \gamma$ events with a photon close to the muon track and remain the dominant background source.

- Background from hadronic decay channels misidentified in the $\tau^{-} \rightarrow \mu^{-} \bar{\nu}_{\mu} \nu_{\tau}(\gamma)$ selection is suppressed by requiring at most two ECAL clusters in the cone, and at most 8 ECAL blocks in the cluster which is associated with the muon track.

- Background from FSR off the $\tau$ is suppressed by requiring that the angle $\theta_{\mu \gamma}$ between the muon track and the photon satisfies $\cos \theta_{\mu \nu}>0.999$.

- In order to remove background from $\mu$ pair events passing the $\tau$ pair selection and entering the $\tau^{-} \rightarrow \mu^{-} \bar{\nu}_{\mu} \nu_{\tau}(\gamma)$ sample, the momentum of the track in the other cone is required to be less than $90 \%$ of the beam energy.

- Finally, all events with muon tracks that lie closer than $0.5^{\circ}$ in $\phi$ to the anode planes of the jet chamber are excluded in order to avoid systematic uncertainties. In this region the reconstructed momenta tend to be less well measured in the data than in the Monte Carlo simulation, and therefore a higher fraction of $\mu$ pair background than expected would enter the $\tau$ sample if tracks near the anode plane were retained.

The $\tau^{-} \rightarrow \mu^{-} \bar{\nu}_{\mu} \nu_{\tau} \gamma$ selection yields 267 events. The selection efficiency is determined using Monte Carlo generated events with full simulation of the OPAL detector [12] based on GEANT [16] to be $(21.2 \pm 0.8) \%$. We have used the Monte Carlo generators KORALZ 4.0 [8], TAUOLA 2.4 [9], and PHOTOS 2.0 [10] to account for radiative corrections. The quoted error includes Monte Carlo statistics and the systematic uncertainties described below. The largest inefficiency apart from the angular acceptance, which cuts away approximately $60 \%$ of the decays, comes from cuts on the number of blocks in the ECAL cluster and the fraction of cluster energy in the most energetic block, which are essential in order to reduce the main background from "muon interactions".

\section{Systematic Studies}

Uncertainties in the measured energy from shower fluctuations, energy resolution, and energy calibration affect the branching fraction measurement because of the $1 \mathrm{GeV}$ photon energy cutoff. These uncertainties influence the selection efficiency and the background from migration across the $1 \mathrm{GeV}$ energy boundary. Accurate modelling of the interaction of muons in the ECAL is crucial for correct reconstruction of the photon energy and for correct estimation of background events from fake photons by "muon-interactions". Further systematic uncertainties are introduced when determining the amount of other background sources from Monte Carlo simulations and the number of $\tau$ decays for the normalisation.

Reference samples of data and Monte Carlo events were compared in order to establish the reliability of the Monte Carlo simulation of the background and the efficiency. Any difference between reference data and Monte Carlo simulation is subsequently taken into account by correction factors obtained from the observed ratio of the two. The total systematic error is taken as the quadratic sum of the Monte Carlo statistical error and the uncertainty in the 
correction factors. The latter is taken as the larger of either half of the deviation of the correction factor from unity or the statistical uncertainty. No correction is applied if reference data and Monte Carlo agree within errors.

The different selection steps are independently investigated for systematic effects.

$\tau$ pair selection: Possible systematic biases in the $\tau$ pair selection are expected to be of the same size as those for the $\mathrm{e}^{+} \mathrm{e}^{-} \rightarrow \tau^{+} \tau^{-}$line shape analysis [17] where a similar selection is used. The systematic uncertainty of approximately $0.5 \%$ from the comparison of data and Monte Carlo simulation constitutes an upper bound on the systematic uncertainty due to the $\tau$ pair selection.

The normalization for the branching fraction, i.e. the total number of $\tau$ pair events, $N_{\tau}$, is obtained from the efficiency and background corrected number of $\mu$ pairs found in the preselected lepton-pair events from which the $\tau$ pairs were selected. The preselected lepton data are a subset of the OPAL data with multihadron, two-photon and cosmic ray rejection but with no explicit $\tau, \mu$ or $e$ selection applied. Generally, the systematic errors on selecting $\mu$ pairs is expected to be smaller than for the selection of $\tau$ pairs because of the clear signature of a $\mu$ pair event. Hence in the present analysis the normalization is done with $\mu$ pair events assuming lepton universality. The systematic errors of the lepton preselection and the $\mu$ pair selection have been studied in detail in [17]. The $\mu$ pair selection efficiency is $(89.3 \pm 1.1) \%$ according to the Monte Carlo. The small corrections which are discussed in [17] are not applied here, but the non- $\mu$ background of $1 \%$ and a systematic uncertainty corresponding to the total of all corrections is taken into account. A total of $120912 \mu$ pair candidates was selected in the data sample which then translates to a total of $133970 \pm 1690$ produced $\tau$ pairs.

Selection of $\tau^{-} \rightarrow \mu^{-} \bar{\nu}_{\mu} \nu_{\tau}(\gamma)$ events: Systematic effects resulting from the likelihood selection of muons are investigated by comparing the efficiency and the purity with reference data. The efficiency of the likelihood selection is checked using $\mathrm{e}^{+} \mathrm{e}^{-} \rightarrow \mu^{+} \mu^{-}$events. Muons from $\mathrm{e}^{+} \mathrm{e}^{-} \rightarrow \mu^{+} \mu^{-}$events are tagged by identifying the track in the opposite cone as a clean muon candidate. Such clean $\mu$ candidates have been selected by requiring exactly one track, which is identified as a muon by the HCAL and muon chambers, with more than $90 \%$ of the beam energy and a small ECAL energy deposit $(<5 \mathrm{GeV})$. The likelihood selection is then applied to the other cone in data and Monte Carlo events. We find agreement in the selection efficiency to within a relative error of $0.3 \%$. This check is restricted to high energy muons only. A further check is performed by determining the branching fraction for the muonic $\tau$ decays using this likelihood selection and comparing it with the OPAL measurement [2]. The branching ratios agree within the relative error of $1.5 \%$.

The purity of the likelihood selection is checked against data by testing the background from hadronic $\tau$ decays passing the likelihood selection. Here the Monte Carlo is compared with the data using a reference sample of hadronic $\tau$ decays selected by requiring a large ratio of ECAL energy to track momentum, indicating that a $\pi^{0}$ is present in the decay, and an invariant mass of the track and photon candidate larger than $500 \mathrm{MeV}$. The fraction of these hadronic $\tau$ decays classified as muonic decays is found to differ between Monte Carlo and reference data by a factor of $1.12 \pm 0.13$, compatible with unity. 
The photon reconstruction: Systematic uncertainties in the third step of the selection, the photon reconstruction, arise from the description of "muon interactions" in the ECAL, the rejection of hadronic $\tau$ decays by ECAL cuts, the photon reconstruction efficiency and the energy calibration for the photons, which affects the energy cut-off and hence the efficiency and background from migration across the energy cut-off.

First, the reliability of the simulation with respect to "muon interactions" in the ECAL has been studied using $\mathrm{e}^{+} \mathrm{e}^{-} \rightarrow \mu^{+} \mu^{-}$events with no photon radiation. Such events are obtained by requiring muon momenta close to the LEP beam energy and a back-to-back event topology. The remaining radiative background with photon energies larger than $200 \mathrm{MeV}$ in the reference sample is $2.5 \%$. These clean muon candidates were examined for the probability to reconstruct fake photons by applying the third step of the $\tau^{-} \rightarrow \mu^{-} \bar{\nu}_{\mu} \nu_{\tau} \gamma$ selection. A fraction of $1.5 \%$ of the muons pass the selection. The background from real photons $(>200 \mathrm{MeV})$ in these events is $31 \%$ according to the simulation and the fraction of muons passing the selection agrees between the data and Monte Carlo simulation within the errors $(0.97 \pm 0.11)$. Because this is the largest background contribution it is also one of the largest contributions to the systematic uncertainty of the reported measurement (c.f. table 2).

Next the influence from hadronic $\tau$ decays on the photon reconstruction was assessed using reference data samples of hadronic $\tau$ decay modes. These reference samples were selected by means of the channel likelihood technique [14] but without using ECAL variables. The deviation between data and Monte Carlo, when applying the third step of the selection procedure to this sample, is statistically significant and results in a correction factor of $1.28 \pm 0.14$ for the background from hadronic $\tau$ decays.

The uncertainty in the knowledge of the photon energy enters into the systematic error due to the $1 \mathrm{GeV}$ cut-off. Here both the efficiency and the background originating from migration depend on the reliability of the reconstructed photon energy. Two effects can cause the photon energy to be mismeasured, the first is mis-calibration of the ECAL, and the second is incorrect estimation of muon energy depositions leading to mis-calculationss of the photon energy in decays where the muon and the photon overlap. A systematic study was performed using $\mathrm{e}^{+} \mathrm{e}^{-} \rightarrow \mu^{+} \mu^{-} \gamma$ events for which the photon direction and its energy can be inferred from the measured muon track momenta. The selection of the events demands that the muon tracks have $z$ chamber hits and that they be separated from the jet chamber anode planes in order to provide a good momentum measurement. Only events with some ECAL energy close to the expected position of the photon are considered in order to ensure correct kinematic reconstruction which is not disturbed by a second, large undetected initial state radiation photon or momentum mismeasurements. This method works best if the photon cluster and muon track have a large opening angle $\left(\theta_{\mu \gamma}>10^{\circ}\right)$ with respect to each other and for photon energies of at least $1 \mathrm{GeV}$. With this reference sample the calibration of the ECAL energy can be tested. For small opening angles $\left(\theta_{\mu \nu}<3.6^{\circ}\right)$ where photon and muon cluster overlap, the uncertainty in the reconstructed photon energy due to the overlapping muon cluster is assessed. For these events, the kinematic reconstruction is less reliable and can only be performed for photon energies above $5 \mathrm{GeV}$. Fig. 4(a) shows the intrinsic biases inherent to this check. For events with large $\theta_{\mu \gamma}$ the kinematically reconstructed photon energy $\left(E_{\text {kinrec }}\right)$ on average deviates from the true energy $\left(E_{\text {true }}\right)$ by at most $100 \mathrm{MeV}$. The rise away from zero at larger $E_{\gamma}$ is due to initial state radiation causing the predicted $E_{\text {kinrec }}$ to be too large. The small $\theta_{\mu \gamma}$ behaviour is dominated by a different bias due to the finite muon momentum resolution, particularly at high muon momenta, and the 
$1 / E_{\gamma}$ behaviour of the $E_{\gamma}$ spectrum. The $E_{\gamma}$ spectrum shape leads to more high energy muons than low energy muons. Therefore, any particular energy bin will, on average, contain more muon events which have migrated from a higher energy bin than from a lower energy bin. This results in a measured muon energy which on average is too low, which in turn results in a kinematically reconstructed photon energy which on average is too high. In the region of large $\theta_{\mu \gamma}$ the expected photon direction is more sensitive to the measured muon momenta. Therefore the requirement that an ECAL cluster lies close to the expected photon direction suppresses the effects of mismeasured muon momenta. Fig. 4(b) shows that reference data events and Monte Carlo events agree within errors when comparing the kinematically reconstructed energy with the measured ECAL energy. Note that in fig. 4(b) $E_{\text {kinrec }}-E_{\text {true }}$ of fig. 4(a) is replaced by $E_{\text {kinrec }}-E_{\mathrm{ECAL}}$ causing a shift downward. However data and Monte Carlo agree in both regions, for large and small angles between muon and photon. Since within the errors of $\simeq 200 \mathrm{MeV}$ the reconstructed photon energy in data and Monte Carlo agree over the entire $E_{\gamma}$ range, we take $200 \mathrm{MeV}$ as the uncertainty at the $1 \mathrm{GeV}$ cut-off energy and account for it in the contributions to the systematic error from migration effects as well as from the selection efficiency.

\begin{tabular}{|c|c|c|}
\hline $\begin{array}{l}\tau^{-} \rightarrow \mu^{-} \bar{\nu}_{\mu} \nu_{\tau} \gamma \text { candidates } N_{\text {cand }} \\
\text { Number of produced } \tau \text {-pairs } N_{\tau}\end{array}$ & \multicolumn{2}{|c|}{$\begin{array}{c}267 \\
133970 \\
\end{array}$} \\
\hline $\begin{array}{l}\text { Selection efficiency } \epsilon_{s e l} \\
\text { uncertainty due to: } \\
\text { reconstructed photon energy } \\
\text { photon reconstruction } \\
\text { photon conversion }\end{array}$ & \multicolumn{2}{|r|}{$\begin{array}{r}+0.3 \% \\
-2.0 \% \\
\pm 1.3 \% \\
\pm 0.3 \% \\
\end{array}$} \\
\hline Background & Number of events & $\begin{array}{l}\text { Correction factor for } \\
\text { Monte Carlo estimate }\end{array}$ \\
\hline $\begin{array}{l}\text { fake photons by muon interaction } \\
\text { final state radiation from the } \tau \\
\text { hadronic } \tau \text { decays } \\
\text { migration across energy cut-off } \\
\mu \text {-bremsstrahlung } \\
\mathrm{e}^{+} \mathrm{e}^{-} \rightarrow \mu^{+} \mu^{-} \\
\mathrm{e}^{+} \mathrm{e}^{-} \rightarrow \mathrm{e}^{+} \mathrm{e}^{-} \mu^{+} \mu^{-} \\
\mathrm{e}^{+} \mathrm{e}^{-} \rightarrow \mathrm{e}^{+} \mathrm{e}^{-} \tau^{+} \tau^{-}\end{array}$ & $\begin{array}{r}68.2 \pm 8.4 \\
21.5 \pm 2.5 \\
44.2 \pm 8.3 \\
11.9 \pm 10.8 \\
0.2 \pm 0.2 \\
5.0 \pm 2.1 \\
0.0 \pm 1.7 \\
0.0 \pm 1.3\end{array}$ & $\begin{aligned} 0.97 & \pm 0.11 \\
1.00 & \pm 0.06 \\
(1.28 \pm 0.14) & \times(1.12 \pm 0.13) \\
& - \\
& - \\
1.00 & \pm 0.06 \\
& - \\
& -\end{aligned}$ \\
\hline Total background $N_{b k g d}$ & \multicolumn{2}{|c|}{$\begin{array}{ll}151.0 & +16.3 \\
- & -13.0\end{array}$} \\
\hline
\end{tabular}

Table 1: Selection efficiency and background estimate. The second column of the background entries shows the Monte Carlo estimate already corrected by the correction factor of column three derived from a comparison with reference data samples (see text). Note that the correction is only applied when the correction factor does not agree with unity within its error.

Finally, the photon reconstruction efficiency derived from the Monte Carlo is tested, again using events of the type $\mathrm{e}^{+} \mathrm{e}^{-} \rightarrow \mu^{+} \mu^{-} \gamma$, requiring $E_{\gamma}>5 \mathrm{GeV}$ and a small opening angle between photon and muon $\left(\theta_{\mu \gamma}<3.6^{\circ}\right)$. Applying the selection cuts of the photon reconstruction to both reference data and Monte Carlo events, the resulting efficiency ratio is shown in fig. 5 as a function of $E_{\gamma}$. The average is $0.96 \pm 0.06$ consistent with unity and the distribution is consistent with being flat in $E_{\gamma}$. The uncertainty in this number is taken to apply down to 

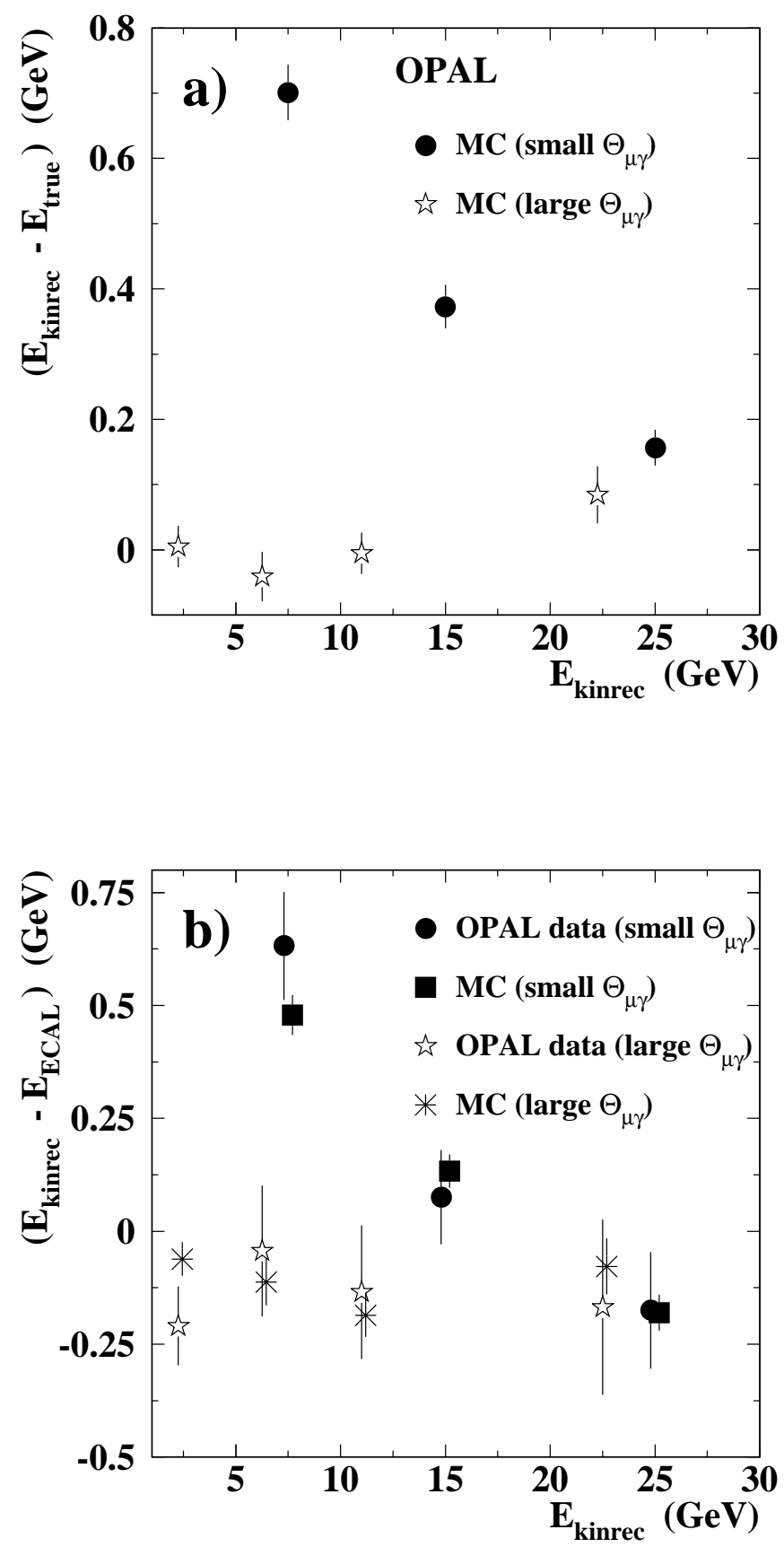

Figure 4: Test of the reconstructed photon energy with $\mathrm{e}^{+} \mathrm{e}^{-} \rightarrow \mu^{+} \mu^{-} \gamma$ events. In (a) the difference between kinematically reconstructed and true photon energy is plotted as a function of $E_{\text {kinrec }}$ for $M C$ events using samples with small $\left(\theta_{\mu \gamma}<3.6^{\circ}\right)$ and large $\left(\theta_{\mu \nu}>10^{\circ}\right)$ opening angles between photon and muon, respectively. In (b) the difference between kinematically reconstructed and measured photon energy in the ECAL is shown against $E_{\text {kinrec }}$ for data and MC. Two regions are considered: small angles between muon and photon where one is sensitive to systematic effects in the reconstructed photon energy due to the overlapping muon, and large angles where the ECAL calibration can be tested. 


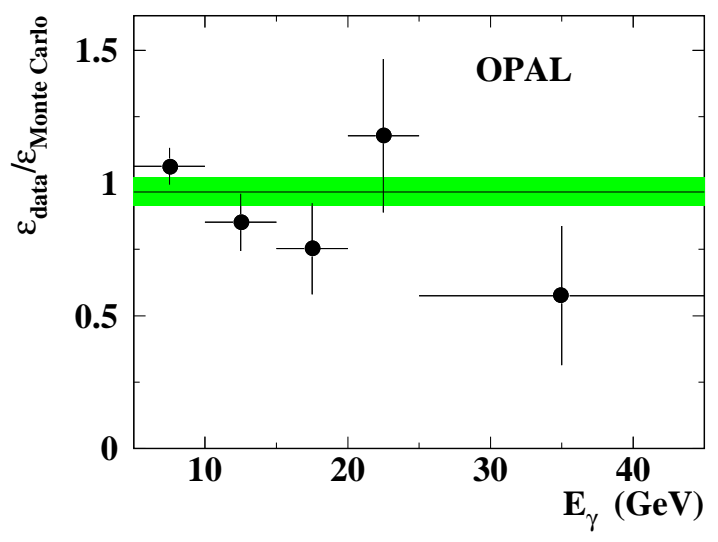

Figure 5: Ratio of the efficiency of the photon reconstruction between reference data and Monte Carlo. The horizontal line indicates the weighted mean of the efficiency ratios in the different energy bins (0.96土0.06).

\begin{tabular}{|l|c|}
\hline Source & $\begin{array}{c}\text { Systematic uncertainty } \\
\text { relative to } \mathrm{B}\left(\tau^{-} \rightarrow \mu^{-} \bar{\nu}_{\mu} \nu_{\tau} \gamma\right)\end{array}$ \\
\hline$\tau$ pair preselection & $\pm 0.5 \%$ \\
Total number of $\tau$ pairs for normalization & $\pm 2.6 \%$ \\
Selection efficiency & $\pm 3.7 \%$ \\
Monte Carlo statistics & $\pm 6.0 \%$ \\
uncertainty due to photon reconstruction & $\pm 0.5 \%$ \\
uncertainty due to photon conversion & $+10.5 \%$ \\
uncertainty on efficiency and migration & $-9.3 \%$ \\
background due to photon energy reconstruction & $\pm 7.2 \%$ \\
Background & $\pm 7.2 \%$ \\
fake photons by muon interaction & $\pm 2.1 \%$ \\
hadronic $\tau$ decays & $\pm 1.8 \%$ \\
final state radiation & $\pm 16.8 \%$ \\
$\mathrm{e}^{+} \mathrm{e}^{-} \rightarrow \mu^{+} \mu^{-}$ & \\
\hline Total systematic error & \\
\hline
\end{tabular}

Table 2: Contributions to the systematic error of the branching fraction measurement.

energies of $1 \mathrm{GeV}$ and is included in the systematic error. The same systematic uncertainty is assumed for background originating from FSR off the $\tau$ because the same photon reconstruction is applied. Theoretical errors in the description of FSR of the $\tau$ production are expected to be much smaller than the assigned error from the photon reconstruction and the statistical error of the Monte Carlo estimate.

The selection efficiency is also influenced by photon conversions due to the requirement that there be only one charged track in the decay. The systematic uncertainty in the simulation of the conversion probability of photons is studied using $\mathrm{e}^{+} \mathrm{e}^{-} \rightarrow \mathrm{e}^{+} \mathrm{e}^{-}$and $\mathrm{e}^{+} \mathrm{e}^{-} \rightarrow \mu^{+} \mu^{-}$ 
events. Tagging such events by identifying one cone as an $e$ or $\mu$ respectively, the conversion rate is compared between data and Monte Carlo by comparing the number of events with more than one good charged track in the other hemisphere. The next-to-highest momentum track is required to have a momentum larger than $0.5 \mathrm{GeV}$ because the test should be restricted to photons with an energy greater than $1 \mathrm{GeV}$. Assuming that the total number of FSR photons in these events is simulated correctly, this directly tests the fraction of converted photons. This comparison results in an uncertainty of $10 \%$ for the conversion rate. Given a conversion rate of $\simeq 5 \%$ this translates into a systematic uncertainty of $0.5 \%$ for the selection efficiency.

Non- $\tau$ background from $\mathrm{e}^{+} \mathrm{e}^{-} \rightarrow \mu^{+} \mu^{-}$events enters into the systematic error only due to the modelling of "muon interactions" and photon reconstruction which have an estimated uncertainty as described previously of $11 \%$ and $6 \%$, respectively. Effects in the $\tau$ pair event selection are negligible in comparison ${ }^{4}$.

Contributions from other background sources are taken directly from the Monte Carlo simulation and their quoted systematic error is from Monte Carlo statistics only. The background contributions and efficiency, together with their systematic uncertainties, are listed in table 1. Table 2 lists the contributions of the various sources to the systematic error of the branching fraction measurement.

\section{Result and Conclusions}

The branching fraction is calculated using

$$
B\left(\tau^{-} \rightarrow \mu^{-} \bar{\nu}_{\mu} \nu_{\tau} \gamma\right)=\frac{N_{c a n d}-N_{b k g d}}{N_{\tau} \epsilon_{s e l} \epsilon_{b o o s t}} .
$$

The number of selected candidate events $N_{\text {cand }}$, the estimated number of background events $N_{b k g d}$, the total number of $\tau$ decays $N_{\tau}$, as well as the selection efficiency $\epsilon_{s e l}$, are given in table 1. The factor $\epsilon_{\text {boost }}$ is needed in order to convert the measured fraction of $\tau^{-} \rightarrow \mu^{-} \bar{\nu}_{\mu} \nu_{\tau} \gamma$ decays with photon energies $\left(E_{\gamma}\right)$ above $1 \mathrm{GeV}$ in the laboratory into a branching fraction for photon energies $\left(E_{\gamma}^{*}\right)$ larger than the corresponding cut-off energy of $20 \mathrm{MeV}$ in the $\tau$ rest frame. This is the minimum energy in the $\tau$ rest frame required for photons to pass the $1 \mathrm{GeV}$ energy cut in the laboratory. Assuming Standard Model energy and angular distributions $\epsilon_{\text {boost }}$ is determined to be $(67.3 \pm 0.2) \%$. The quoted error arises from Monte Carlo statistics only.

Using the numbers from table 1 , the resulting branching fraction for $\tau^{-} \rightarrow \mu^{-} \bar{\nu}_{\mu} \nu_{\tau} \gamma$ is measured to be:

$$
B\left(\tau^{-} \rightarrow \mu^{-} \bar{\nu}_{\mu} \nu_{\tau} \gamma\right)=(3.0 \pm 0.4 \pm 0.5) \times 10^{-3} \quad \text { for } \quad E_{\gamma}^{*}>20 \mathrm{MeV}
$$

where the first error is statistical and the second systematic. The result is in good agreement with the theoretical expectation of $2.82 \times 10^{-3}$ taken from the Monte Carlo with leading-log calculation as implemented in PHOTOS[10]. Fig. 6 shows the comparison between data and Monte Carlo for the measured photon energy spectrum (a), the distribution of the angle between

\footnotetext{
${ }^{4}$ Large systematic uncertainties in the $\mathrm{e}^{+} \mathrm{e}^{-} \rightarrow \mu^{+} \mu^{-}$background occur in the anode plane regions[2] which are excluded in this analysis.
} 

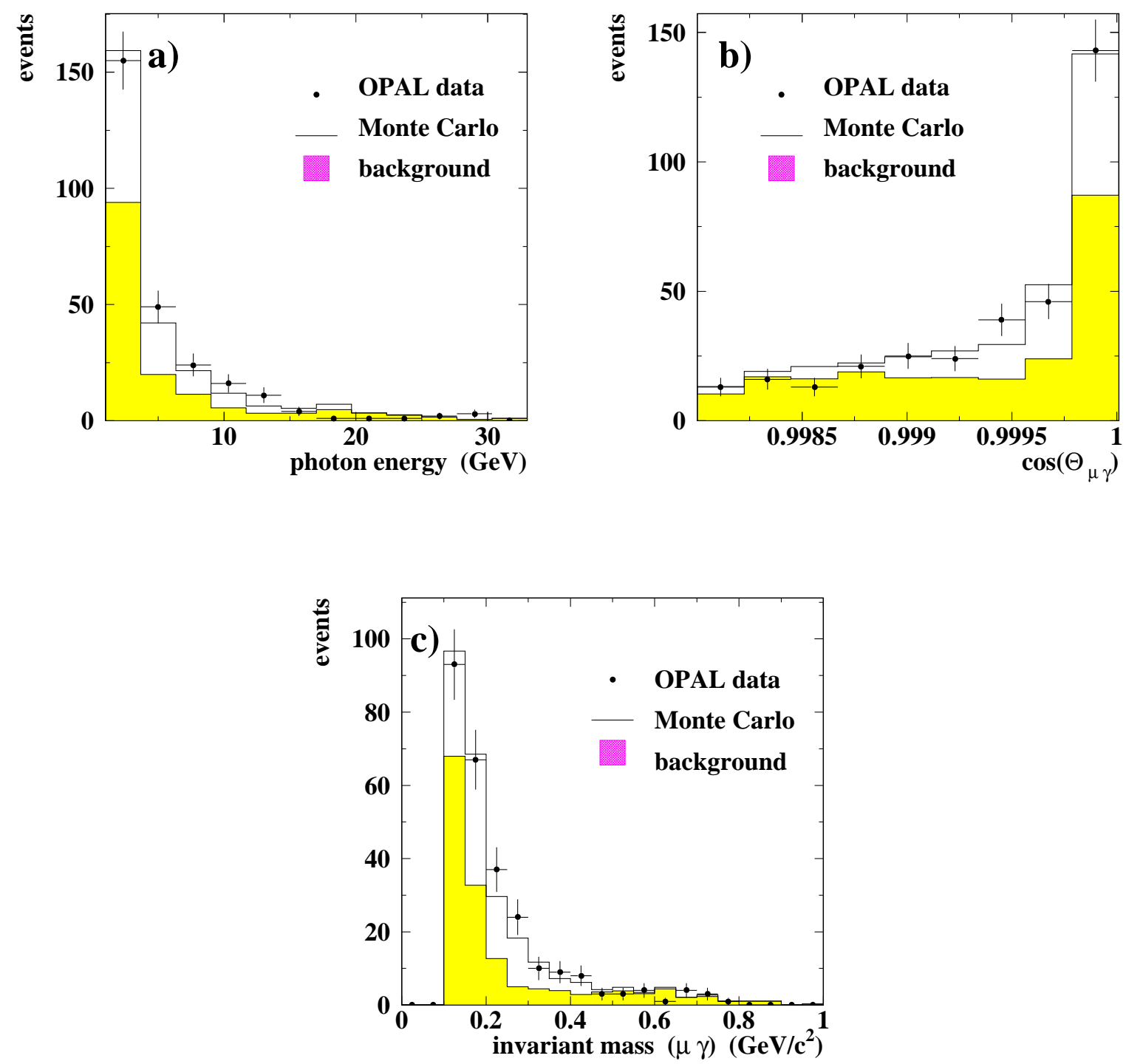

Figure 6: Comparison between data and Monte Carlo distributions for the decay $\tau^{-} \rightarrow \mu^{-} \bar{\nu}_{\mu} \nu_{\tau} \gamma$. (a) the photon energy spectrum, (b) the photon angular distribution, and (c) the reconstructed $\mu \gamma$ invariant mass. The points with errors show the $\tau^{-} \rightarrow \mu^{-} \bar{\nu}_{\mu} \nu_{\tau} \gamma$ candidates obtained from the data and the solid histograms indicate the Monte Carlo estimates. Background is indicated by the shaded histograms. In (b) the cut on $\cos \theta_{\mu \gamma}$ has been removed. 
muon and photon (b), and the $\mu \gamma$ invariant mass (c). The good description of the measured data by the prediction gives further confidence in the procedure for background estimates using the Monte Carlo together with reference samples. In particular, the first and second bin of the $\mu \gamma$ invariant mass distribution shows this, as the background peaks in the first bin whereas the signal, i.e. data minus background, has its maximum in the second bin.

In order to compare our result with the measurement from MARK II [11] we also analyze the branching fraction for cut-off energies of $E_{\gamma}^{*}>37 \mathrm{MeV}$. This results in a value of $B\left(\tau^{-} \rightarrow \mu^{-} \bar{\nu}_{\mu} \nu_{\tau} \gamma\right)=(2.7 \pm 0.6) \times 10^{-3}$ for $E_{\gamma}^{*}>37 \mathrm{MeV}$, in excellent agreement with the MARK II measurement, but with an error smaller by a factor of two.

\section{Acknowledgements}

We particularly wish to thank the SL Division for the efficient operation of the LEP accelerator and for their continuing close cooperation with our experimental group. In addition to the support staff at our own institutions we are pleased to acknowledge the

Department of Energy, USA,

National Science Foundation, USA,

Particle Physics and Astronomy Research Council, UK,

Natural Sciences and Engineering Research Council, Canada,

Israel Ministry of Science,

Israel Science Foundation, administered by the Israel Academy of Science and Humanities, Minerva Gesellschaft,

Japanese Ministry of Education, Science and Culture (the Monbusho) and a grant under the Monbusho International Science Research Program,

German Israeli Bi-national Science Foundation (GIF),

Direction des Sciences de la Matière du Commissariat à l'Energie Atomique, France,

Bundesministerium für Bildung, Wissenschaft, Forschung und Technologie, Germany,

National Research Council of Canada,

Hungarian Foundation for Scientific Research, OTKA T-016660, and OTKA F-015089. 


\section{References}

[1] Particle Data Group, Phys. Rev. D 50 (1994) 1173;

DELPHI Collaboration, P. Abreu et al., Phys. Lett. B 357 (1995) 715;

ALEPH Collaboration, D. Buskulic et al., Z. Phys. C 70 (1996) 561.

[2] OPAL Collaboration, R. Akers et al., Z. Phys. C 66 (1995) 543.

[3] OPAL Collaboration, G. Alexander et al., Phys. Lett. B 369 (1996) 163.

[4] See for example, H.S. Chen, Proceedings of the XVII Int. Symp. on Lepton-Photon Interactions, Beijing, China, August 10-15, 1995.

[5] C. Fronsdal, H. Überall, Phys. Rev. 113 (1959) 654;

R.E. Behrends, R.J. Finkelstein, A. Sirlin, Phys. Rev. 101 (1956) 866.

[6] W. Eichenberger, R. Engfer, A. van der Schaaf, Nucl. Phys A 412 (1984) 523.

[7] A. Stahl, H. Voss, Bonn Univ. preprint BN-HE-96-02 (1996), submitted to Z. Phys. C.

[8] S. Jadach, B.F.L. Ward, Z. Wạs, Comp. Phys. Comm. 79 (1994) 503.

[9] S. Jadach, Z. Wa̧s, R. Decker, J.H. Kühn, Comp. Phys. Comm. 76 (1993) 361.

[10] E. Barberio, Z. Wạs, Comp. Phys. Comm. 79 (1994) 291.

[11] D.Y. Wu et al., Phys. Rev. D 41 (1990) 2339.

[12] OPAL Collaboration, K. Ahmet et al., Nucl. Inst. Meth. A 305 (1991) 275;

P. P. Allport et al, Nucl. Inst. Meth. A 324 (1993) 34;

P. P. Allport et al, Nucl. Inst. Meth. A 346 (1994) 476.

[13] OPAL Collaboration, M.Z. Akrawy et al., Phys. Lett. B 247 (1990) 458.

[14] OPAL Collaboration, R. Akers et al., Z. Phys. C 66 (1995) 31.

[15] OPAL Collaboration, R. Akers et al., Z. Phys. C 63 (1994) 181.

[16] J. Allison et al., Nucl. Inst. Meth, A 317 (1992) 47;

R. Brun et al., GEANT3 Users Guide, CERN DD/EE/84-1 (1989).

[17] OPAL Collaboration, R. Akers et al., Z. Phys. C 61 (1994) 19. 